\title{
Association between cardiometabolic health and objectively- measured, free-living sleep parameters in a rural African setting: a pilot study.
}

lan Cook ( $\square$ ian.cook@ul.ac.za)

University of Limpopo Faculty of Humanities https://orcid.org/0000-0001-5098-2936

Matlawa Mohlabe

University of Limpopo Faculty of Health Sciences

Herbert Mabalane Makgopa

University of Limpopo Faculty of Health Sciences

\section{Research note}

Keywords: insulin resistance, metabolic syndrome, anthropometry, accelerometer, actigraphy, movement monitor, measurement

Posted Date: June 1st, 2020

DOI: https://doi.org/10.21203/rs.3.rs-31984/v1

License: (c) (i) This work is licensed under a Creative Commons Attribution 4.0 International License. Read Full License 


\section{Abstract}

Objectives To investigate the relationship between objectively-measured, free-living sleep quantity and quality, and cardiometabolic health, in a rural African setting in 139 adults ( $\geq 40$ years, female: $n=99$, male: $n=40$ ). Wrist-mounted, tri-axial accelerometry data was collected over nine days. Measures of sleep quantity and quality, and physical activity were extracted from valid minute-by-minute data. Self-reported data included behavioural, health and socio-demographic variables. Biological data included body composition, resting blood pressure and fasting blood glucose, insulin and lipids. Regression models were constructed with insulin resistance (IR), Cardiometabolic (CM) risk and a metabolic z-score, as dependent variables, adjusting for socio-demographic, behavioural and biological factors. Results Nocturnal sleep time was longer in females $(p=0.054)$ and sleep quality was better in males $(p \leq 0.017)$. Few participants slept $>9$ hours/night (4-5\%), and $46-50 \%$ slept $<7$ hours/night. IR and CM risk was higher in females $(p \leq 0.006)$. In adjusted models, sleep variables were independently associated with $I R(p<0.05)$. Sleep quantity was linearly $(p<0.05)$ and non-linearly $(p \leq 0.0196)$ associated with IR, and non-linearly $(p \leq 0.0398)$ associated with CM risk. Sleep quality was linearly related with $I R(p<0.05)$. A number of non-sleep behavioural variables were independently associated with $\mathrm{CM}$ risk (alcohol and tobacco use, $\mathrm{p} \leq 0.034$ ) and IR (physical activity, sugarsweetened beverage consumption, $p<0.05)$.

\section{Introduction}

Sleep health is closely linked to metabolic health [1], and while there is extensive literature from industrialised settings [2], there is a paucity of data from low and middle-income countries, especially free-living, objective measures of sleep [3]. Within the South African context, self-reported sleep duration is associated with cardiometabolic health in mainly urban settings [4,5]. Given the lack of objectively-measured, free-living sleep parameters in any South African setting, the objective of this study was to use wrist-actigraphy to investigate the association between sleep parameters and cardiometabolic health in a rural African setting during a cross-sectional survey, and thus extend the findings of self-report sleep duration and cardiometabolic health [4-6]

\section{Methods}

Dikgale Health and Demographic Surveillance System site (DHDSS) sample

A convenience sample of 167 adults was recruited from the DHDSS site [7]. These participants formed part of a larger study cohort ( $\geq 40$ years) $[6,8]$. Trained field workers collected self-reported and measured data from participants by means of questionnaires, anthropometry, oscillometric blood pressure measurement, ultrasound scans, and venipuncture $[6,8]$. We calculated body mass index $\left(\mathrm{BMI}, \mathrm{kg} / \mathrm{m}^{2}\right)$, and Conicity Index $(\mathrm{Cl})[9]$. Questionnaire data included behavioural, health and socio-demographic variables [6, 8]. Nine day, free-living, wrist-mounted accelerometry data was collected [10]. The ultrasound scans were not considered for this analysis.

\section{Blood sample collection and analysis}

A registered nurse collected fasting blood samples. The samples were analysed centrally; procedures and calculations are described in detail elsewhere [8]. The Homeostasis Model Assessment of Insulin Resistance (HOMA-IR) was calculated from fasting blood glucose and insulin [11].

\section{Criteria for Metabolic Syndrome}

In accordance with the harmonized Joint Interim Statement (JIS) definition [12], the presence of the Metabolic Syndrome (MetS) required three of the following components, with waist circumference not a prerequisite: elevated waist circumference (WC): females $\geq 92 \mathrm{~cm}$, males $\geq 86 \mathrm{~cm}$; elevated triglycerides (TG): $\geq 1.7 \mathrm{mmol} / \mathrm{l}$; reduced high-density lipoprotein cholesterol (HDL-C): men $<1.0 \mathrm{mmol} / \mathrm{l}$, women $<1.3 \mathrm{mmol} /$; elevated resting blood pressure $\geq 130 / 85 \mathrm{mmHg}$ or on hypertension treatment; and elevated fasting glucose (GC) $\geq 5.6$ $\mathrm{mmol} / \mathrm{I}$ or on diabetes treatment. For this study population-specific WC cut-points were chosen [13].

Using the five criteria from the JIS definition for MetS (JIS-MetS), we calculated sex-specific z-scores for HDL-C, TG, GC, WC and MAP (Mean Arterial Pressure), which were summed to create a MetS z-Score (MetSz) [14].

\section{Accelerometer data collection and data reduction}

Participants wore a small, light-weight, wrist-worn tri-axial accelerometer for 9 days $(4.6 \mathrm{~cm} \times 3.3 \mathrm{~cm} \times 1.5 \mathrm{~cm}, 19 \mathrm{grams}$, ActiGraph wGT3XBT, Actigraph, LLC, Pensacola, FL, 2013) [15-18]. The preparation, initialization, mounting, data processing and extraction of physical activity (PA) and sleep parameters are described in detail elsewhere [10]. 
Vector Magnitude (VM)- and Ambulation-defined PA variables were defined as counts/day and steps/day, respectively [19]. Sleep indices included Total Sleep Time (TST), Nocturnal Sleep Time (NST), Sleep Efficiency (SE), Wake After Sleep Onset (WASO), Activity Counts during sleep (AC), and Sleep Fragmentation Index (SFI) [20-22]. Nocturnal periods were defined as 18h01-05h59. Sufficient sleep quantity and quality was defined as 7-9 hours [23] and $\geq 85 \%$ [24], respectively.

\section{Statistical analyses}

Descriptive statistics comprised means (one standard deviation), medians (inter-quartile range) and frequencies. Relationships between categorical variables were examined through Fisher's Exact Test. For continuous data, independent $t$ tests examined differences between sexes and risk groups. Where required a non-parametric test was employed. Bi-variate relationships were examined using linear regression.

Forced multiple linear regression models examined the relationship between HOMA-IR and MetSz, and sleep quantity and quality variables, adjusting for socio-demographic, behavioural and biological variables. A measure of adiposity (Cl) was included in the HOMAIR model but not the MetSz model, because WC formed part of the dependent variable. Non-normally distributed, continuous variables were either transformed to categorical variables (quantiles) or log transformed. Models assumptions were examined [25].

Forced binary logistic regression models examined the relationship between MetS risk (low/high) according to the JIS definition, and tertiles (Q1=low/Q2/Q3=high) of sleep quantity and quality variables, adjusting for socio-demographic, behavioural and biological variables. Anthropometry was not included as an independent variable because WC formed part of the JIS risk definition.

Forced ordinal logistic regression models examined the relationship between HOMA-IR levels (tertiles), and tertiles of sleep quantity and quality variables, adjusting for socio-demographic, behavioural and biological variables, including the level of adiposity (CI). Regression coefficients for binary and ordinal logistic regression models were expressed as odds ratios (OR $\pm 95 \%$ confidence intervals).

Goodness of fit criteria included Akaike's Information Criteria (AIC) (all models), adjusted or pseudo $\mathrm{R}^{2}$ (all models), Hosmer-Lemeshow test (Binary logistic) and Area Under the Curve (AUC) (Binary Logistic).

Post-hoc contrasts were run for binary and ordinal logistic regression models to test for linear and non-linear trends, and pairwise comparison of groups (Bonferroni correction).

Data were analysed using appropriate statistical software (Stata/SE for Windows: Release 15.1. College Station, TX: StataCorp LP, 2020). Significance for all inferential statistics was set at $p<0.05$.

\section{Results}

Of the 167 raw accelerometry data files, 157 had valid data for at least one weekday and one weekend day. Once combined with the questionnaire and biological data, 139 participants had complete data.

Females consumed more SSB (sugar-sweetened beverages), experienced poorer sleep quality, and were more physically active and insulin resistant (Table 1, $\mathrm{p} \leq 0.07$ ). Males were leaner, used more alcohol and tobacco products and experienced lower people-to-bedroom density $(\mathrm{p} \leq 0.040)$. The JIS high risk group had a higher SES, a lower HIV+ prevalence, were mostly obese, more insulin resistant, and likely to be in a partnered relationship (Table $1, p \leq 0.040$ ).

In bi-variate analysis, sex, SES, Cl, WC, BMI, SSB and WASO were significantly associated with HOMA-IR ( $\mathrm{p} \leq 0.05)$. SES, BMI, Cl, WC and HIV status were significantly related to MetSz ( $\mathrm{p} \leq 0.027)$.

Except for the MetSz models (Table 2), all other models were significant (Table 2, Figure 1A-F, Figure S1A-F - see Additional file 1, $\mathrm{p} \leq 0.0203$ ). The binary logistic regression models (Figure 1A-C, Figure S1A-C - see Additional file 1) showed good fit (Hosmer-Lemeshow: $p \geq 0.3744$; AUC: 0.808-0.877).

Poor sleep quality was significantly associated with poor metabolic health (increasing HOMA-IR levels) $(p<0.05)$, while TST was significantly and directly related to HOMA-IR $(p<0.05)$ (Table 2). Males tended to have lower HOMA-IR, and a higher SES was associated with a higher HOMA-IR $(p<0.05)$. SSB was significantly and directly associated with HOMA-IR $(p<0.05)$. Increasing levels of PA tended to be associated with reduced levels of HOMA-IR. Only in one model did sleep quantity and quality parameters concurrently and significantly contribute to explaining the variance in HOMA-IR (Table 2). 
The MetSz models were not associated with any sleep variable (Table 2). While sex was not significant, HIV+ status was quite consistently associated with lower (healthier) MetSz. As with the HOMA-IR model, SES status was positively associated with a higher (poorer) MetSz (Table 2).

There was a significant non-linear trend (U-shaped) between JIS-MetS risk and NST ( $p=0.0196)$ (Figure 1A), such that for NST Q2, there was $80 \%$ less likelihood of being at high risk for JIS-MetS. There was also a significant difference in frequencies between NST Q1 and Q2 $(p=0.021)$ (Figure 1A).

There was a significant positive, linear trend between JIS-MetS risk and SFI ( $p=0.0001$ ) (Figure 1C). The odds of high risk JIS-MetS for SFI Q3 was high $(\mathrm{OR}=69.81,8.44 ; 577.63)$, however the confidence intervals were wide. There were significant differences between the frequencies for Q3 versus Q1 and Q2 ( $<<0.001)$ (Figure 1C).

Being a current user of both alcohol and tobacco products carried a significant higher odds for JIS-MetS high risk in the NST (OR=4.51, $\mathrm{p}=0.034)$ and $\mathrm{SFI}(\mathrm{OR}=6.55, \mathrm{p}=0.023)$ models (Figure $1 \mathrm{~A}-\mathrm{C})$.

In binary logistic regression models (Figure 1A-C) males had significantly lower odds for JIS-MetS high risk (OR $\approx 0.15, \mathrm{p} \leq 0.006)$, and similarly for HIV+ in the NST and SFI models (OR=0.25, $\mathrm{p} \leq 0.0035)$. Once adjusted for adiposity $(\mathrm{Cl})$, sex was no longer a significant factor ( $p \geq 0.097)$. However, HIV+ status remained a significant factor in most models $(O R \approx 0.26, p \leq 0.048)($ Figure $1 A-C)$.

There was a significant linear trend between TST and HOMA-IR levels ( $p=0.0444$ ) (Figure 1D). Compared to TST Q1 and Q2, being in TST Q3 (longest sleep time) increased the odds of being in the highest HOMA-IR level (Q3) by a factor of 2.84 ( $p=0.044)$ (Figure 1D).

There were significant linear relationships between SE and WASO, and HOMA-IR ( $\mathrm{p} \leq 0.007)$ (Figure 1E). A high SE (Q3) was 85\% less likely to result in HOMA-IR Q3 ( $p=0.001)$. Being in WASO Q2 and Q3 significantly increased the odds for HOMA-IR Q3; OR=3.17 and $\mathrm{OR}=6.75$, respectively $(\mathrm{p} \leq 0.019)$.

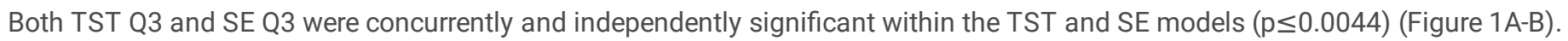

SFI and AC were significantly associated with increasing HOMA-IR levels ( $\mathrm{p} \leq 0.0201)$ (Figure 1F). Being in SFI Q2 and Q3 significantly increased the odds for HOMA-IR Q3, OR=4.67 ( $p=0.004)$ and OR=10.91 ( $p<0.001)$, respectively. For AC Q3 the odds of being in the HOMAIR Q3 was $3.01(\mathrm{p}=0.020)$ (Figure 1F).

In ordinal logistic regression models (Figure 1D-F), being in the VM Q2 level, significantly decreased the odds of being in HOMA-IR Q3 by a factor of $\approx 0.31$ ( $\mathrm{p} \leq 0.039$ ). In contrast, for both $\mathrm{Cl} \mathrm{Q} 2$ and Q3 levels, the odds of being in HOMA-IR Q3 were significantly increased; Q2 $\mathrm{OR} \approx 2.9$ and $\mathrm{Q} 3 \mathrm{OR} \approx 3.79$, respectively $(\mathrm{p} \leq 0.045)$ (Figure 1D-F).

Expressing sleep quantity and quality parameters in terms of sleep health guidelines, we found significant non-linear associations with JIS-MetS risk ( $p \leq 0.0308$ ) (Figure S1A-F, see Additional file 1). The relationship between sleep categories for both TST and NST, and JISMetS risk were U-shaped, the nadir at 7-9 hours of sleep (Figure S1A-B, see Additional file 1).

In binary logistic regression models (Figure S1A-C, see Additional file 1) males had significantly lower odds for JIS-MetS high risk $(O R \approx 0.14, p=0.004)$, and similarly for $\mathrm{HIV}+(\mathrm{OR} \approx 0.26, p \leq 0.0034)$. Once adjusted for adiposity $(\mathrm{Cl})$, sex was no longer a significant factor $(p \geq 0.121)$. However, HIV+ status remained a significant factor in most models $(O R \approx 0.23, p \leq 0.045)$ (Figure S1A-C, see Additional file 1$)$.

In ordinal logistic regression models (Figure S1D-F, see Additional file 1) physical activity (VM Q2) significantly reduced the odds of being in HOMA-IR Q3 by a factor of $\approx 0.26$ ( $\mathrm{p} \leq 0.010)$. For both CI Q2 and Q3 levels, the odds of being in HOMA-IR Q3 were significantly increased (Q2 OR $\approx 3.16, \mathrm{Q} 3 \mathrm{OR} \approx 3.89, \mathrm{p} \leq 0.022$ ). For $\mathrm{SE} \geq 85 \%$, there was a 76\% lower likelihood of being in HOMA-IR Q3. Significantly more participants were classified as low HOMA-IR (Q1) in the SE $\geq 85 \%$ category (Figure S1D, see Additional file 1).

\section{Discussion}

This analysis is novel in that, as far as the authors are aware, this is the first free-living, actigraphy-measured sleep and cardiometabolic health study from a South African setting.

The main findings of this analysis were first that sleep quality and quantity measures were independently associated with HOMA-IR, and to lesser extent JIS-MetS. Second, we found non-linear (U-shaped) relationships between categories of sleep quantity and cardiometabolic risk, for both HOMA-IR and JIS-MetS. Third, all sleep quality measures were consistently associated with especially 
HOMA-IR. Fourth, no sleep parameter was associated with the continuous MetSz score. Fifth, a number of socio-demographic-, non-sleep behavioural-, and biological factors were independently associated with HOMA-IR and JIS-MetS.

Our findings are in agreement with the linear relationship between sleep duration and HOMA-IR in black, urban women, although we found far more women had short sleep time compared with self-report measures [4]. A recent study from the METS group found long sleep duration in a black, urban South African sample [5].

Unadjusted sleep quantity did not differ significantly across the sexes $(p=0.054)$, which is on contrast to self-report measures [4]. Sleep quality was poorer in females, although sex did not reach significance in most HOMA-IR models.

In contrast with some self-reported PA studies [4], we found PA volume to be significantly, inversely related to HOMA-IR, but not associated with JIS-MetS risk as in other self-report studies [5].

In conclusion, we report the significant, independent importance of objectively-measured sleep quantity and quality in relation to cardiometabolic health in a rural South African setting.

\section{Limitations}

Due to the small sample size and cross-sectional, convenience sampling in this study, the results cannot be readily generalized, nor can causality be shown.

\section{Abbreviations}

BMI: body mass index

Cl: Conicity Index

DHDSS: Dikgale Health and Demographic Surveillance System

GC: Glucose

HDL-C: High-density Lipoprotein Cholesterol

HIV: Human Immunodeficiency Virus

HOMA-IR: Homeostasis Model Assessment of Insulin Resistance

JIS: Joint Interim Statement

LDL-C: Low-density Lipoprotein Cholesterol

MAP: Mean Arterial Pressure

MetS: Metabolic Syndrome

MetSz: Metabolic Syndrome z-score

NST: Nocturnal Sleep Time

PA: Physical Activity

SE: Sleep Efficiency

SES: Socio-Economic Status

SFI: Sleep Fragmentation Index

SSB: Sugar sweetened beverages

TC: Total Cholesterol 
TG: Triglyceride

TST: Total Sleep Time

VM: Vector Magnitude

WASO: Wake After Sleep Onset

WC: Waist circumference

\section{Declarations}

Ethics approval and consent to participate

Ethics approval for the AWI-Gen Phase 1 survey data and the accelerometer data collection was obtained from the Medunsa Research and Ethics Committee of the University of Limpopo (MREC/HS/195/2014:CR). The participants recruited into the original studies were informed about the study objectives, expected outcomes, benefits and the risks associated with it. Written informed consent was obtained from the participants prior to interviews and measurements.

Availability of data and material

The dataset analysed during the current study is available from the corresponding author on reasonable request.

Funding

The AWI-Gen Collaborative Centre is funded by the National Human Genome Research Institute (NHGRI), Office of the Director (OD), Eunice Kennedy Shriver National Institute Of Child Health \& Human Development (NICHD), the National Institute of Environmental Health Sciences (NIEHS), the Office of AIDS research (OAR) and the National Institute of Diabetes and Digestive and Kidney Diseases (NIDDK), of the National Institutes of Health (NIH) under award number U54HG006938 and its supplements, as part of the H3Africa Consortium. Additional funding was leveraged from the Department of Science and Technology, South Africa, award number DST/CON 0056/2014, and from the African Partnership for Chronic Disease Research (APCDR). The accelerometry section of this study was funded by the Competitive Programme for Rated Researchers, National Research Foundation, South Africa, award number CPRR14080687621.

Acknowledgements

The authors would like to thank the communities, participants and field workers of the Dikgale Demographic and Health Surveillance System Site.

Consent for publication

Not applicable.

Competing interests

The authors declare no competing interests.

Author's contributions

IC was the principal investigator of the accelerometer data on which this manuscript is based, who initiated the research and obtained funding, co-wrote and edited the research proposal, supervised the data entry, analyzed the data and wrote the first draft manuscript. MM co-wrote and edited the research proposal, collected field data, supervised the field work, performed the data entry, commented on the manuscript. HMM commented on- and contributed to the manuscript.

\section{References}

1. Smiley A, Wolter S, Nissan D. Mechanisms of Association of Sleep and Metabolic Syndrome. J Med Clin Res Rev. 2019;3:1-9.

2. Anothaisintawee T, Reutrakul S, Van Cauter E, Thakkinstian A. Sleep disturbances compared to traditional risk factors for diabetes development: systematic review and meta-analysis. Sleep Med Rev. 2016;30:11-24.

Page 6/16 
3. Cole HV, Owusu-Dabo E, Iwelunmor J, Newsome V, Meeks K, Agyemang C et al. Sleep duration is associated with increased risk for cardiovascular outcomes: a pilot study in a sample of community dwelling adults in Ghana. Sleep Med. 2017;34:118-25.

4. Rae DE, Pienaar PR, Henst RHP, Roden LC, Goedecke JH. Associations between long self-reported sleep, obesity and insulin resistance in a cohort of premenopausal black and white South African women. Sleep Health. 2018;4:558-64.

5. Rae DE, Dugas L, Roden L, Lambert EV, Bovet P, Plange-Rhule J et al. Associations between self-reported sleep duration and cardiometabolic risk factors in young African-origin adults from the five-country modeling the epidemiologic transition study (METS). Sleep Health. Forthcoming 2020.

6. Mashinya F, Alberts M, Cook I, Ntuli S. Determinants of body mass index by gender in the Dikgale Health and Demographic Surveillance System site, South Africa. Glob Health Action. 2018;11:1537613.

7. Alberts M, Dikotope SA, Choma SR, Masemola ML, Modjadji SE, Mashinya F et al. Health \& Demographic Surveillance System Profile: The Dikgale Health and Demographic Surveillance System. Int J Epidemiol. 2015;44:1565-71.

8. Ali SA, Soo C, Agongo G, Alberts M, Amenga-Etego L, Boua RP et al. Genomic and environmental risk factors for cardiometabolic diseases in Africa: methods used for Phase 1 of the AWI-Gen population cross-sectional study. Glob Health Action. 2018;11:1507133.

9. Valdez R, Seidell JC, Ahn YI, Weiss KM. A new index of abdominal adiposity as an indicator of risk for cardiovascular disease. A cross-population study. Int J Obes Relat Metab Disord. 1993;17:77-82.

10. Cook I, Mohlabe M, Alberts M. Descriptive epidemiology of objectively-measured, free-living sleep parameters in a rural African setting. BMC Res Notes. Under Review 2020. Available from: https://www.researchsquare.com/article/rs-14209/v1 [Accessed 24 May 2020].

11. Matthews DR, Hosker JP, Rudenski AS, Naylor BA, Treacher DF, Turner RC. Homeostasis model assessment: insulin resistance and beta-cell function from fasting plasma glucose and insulin concentrations in man. Diabetologia. 1985;28:412-9.

12. Alberti KG, Eckel RH, Grundy SM, Zimmet PZ, Cleeman JI, Donato KA et al. Harmonizing the metabolic syndrome: a joint interim statement of the International Diabetes Federation Task Force on Epidemiology and Prevention; National Heart, Lung, and Blood Institute; American Heart Association; World Heart Federation; International Atherosclerosis Society; and International Association for the Study of Obesity. Circulation. 2009;120:1640-5.

13. Motala AA, Esterhuizen T, Pirie FJ, Omar MA. The prevalence of metabolic syndrome and determination of the optimal waist circumference cutoff points in a rural South African community. Diabetes Care. 2011;34:1032-7.

14. Lee JC, Westgate K, Boit MK, Mwaniki DL, Kiplamai FK, Friis H et al. Physical activity energy expenditure and cardiometabolic health in three rural Kenyan populations. Am J Hum Biol. 2019;31:e23199-e.

15. Whitaker KM, Pettee Gabriel K, Jacobs DR, Jr., Sidney S, Sternfeld B. Comparison of Two Generations of ActiGraph Accelerometers: The CARDIA Study. Med Sci Sports Exerc. 2018;50:1333-40.

16. Migueles JH, Cadenas-Sanchez C, Ekelund U, Delisle Nyström C, Mora-Gonzalez J, Löf M et al. Accelerometer data collection and processing criteria to assess physical activity and other outcomes: a systematic review and practical considerations. Sports Med. 2017;47:1821-45.

17. Slater JA, Botsis T, Walsh J, King S, Straker LM, Eastwood PR. Assessing sleep using hip and wrist actigraphy. Sleep Biol Rhythms. 2015;13:172-80.

18. Full KM, Kerr J, Grandner MA, Malhotra A, Moran K, Godoble S et al. Validation of a physical activity accelerometer device worn on the hip and wrist against polysomnography. Sleep Health. 2018;4:209-16.

19. Wennman H, Pietilä A, Rissanen H, Valkeinen H, Partonen T, Mäki-Opas T et al. Gender, age and socioeconomic variation in 24-hour physical activity by wrist-worn accelerometers: the FinHealth 2017 Survey. Sci Rep. 2019;9:6534.

20. Ancoli-Israel S, Martin JL, Blackwell T, Buenaver L, Liu L, Meltzer LJ et al. The SBSM guide to actigraphy monitoring: clinical and research applications. Behav Sleep Med. 2015;13:S4-S38.

21. Chung S, Youn S, Lee C, Jo M-W, Park J, Jo SW et al. Environmental noise and sleep disturbance: night-to-night variability of sleep/wake pattern. Sleep Med Res. 2016;7:78-81.

22. Ko Y, Lee J-Y. Effects of feet warming using bed socks on sleep quality and thermoregulatory responses in a cool environment. J Physiol Anthropol. 2018;37:13.

23. Hirshkowitz M, Whiton K, Albert SM, Alessi C, Bruni O, DonCarlos L et al. National Sleep Foundation's sleep time duration recommendations: methodology and results summary. Sleep Health. 2015;1:40-3.

24. Fung MM, Peters K, Ancoli-Israel S, Redline S, Stone KL, Barrett-Connor E. Total sleep time and other sleep characteristics measured by actigraphy do not predict incident hypertension in a cohort of community-dwelling older men. J Clin Sleep Med. 2013;9:585-91.

Page $7 / 16$ 
25. Mehmetoglu M: REGCHECK: Stata module to examine regression assumptions. In: EconPapers. 2014. https://EconPapers.repec.org/RePEc:boc:bocode:s457943. Accessed 24 May 2020.

\section{Tables}


Table 1 Descriptive statistics of demographic, behavioural and biological characteristics by sex and cardio-metabolic risk

\begin{tabular}{|c|c|c|c|c|c|c|}
\hline & \multicolumn{3}{|c|}{ Sex } & \multicolumn{3}{|c|}{$\begin{array}{c}\text { Cardio-metabolic Risk (JIS Harmonized } \\
\text { definition) }\end{array}$} \\
\hline & $\begin{array}{l}\text { Females } \\
(\mathrm{n}=99)\end{array}$ & $\begin{array}{l}\text { Males } \\
(\mathrm{n}=40)\end{array}$ & $\begin{array}{c}\text { p- } \\
\text { values }\end{array}$ & $\begin{array}{c}\text { Low Risk }(<3 \\
\text { factors }) \\
(\mathrm{n}=90)\end{array}$ & $\begin{array}{c}\text { High Risk } \\
\text { ( } \geq 3 \text { factors) } \\
(\mathrm{n}=49)\end{array}$ & $\begin{array}{c}\text { p- } \\
\text { values }\end{array}$ \\
\hline \multicolumn{7}{|l|}{ Socio-demographic } \\
\hline Age (years) & $\begin{array}{l}52.0 \\
(6.9)\end{array}$ & $\begin{array}{l}53.9 \\
(7.5)\end{array}$ & 0.165 & $51.9(7.4)$ & $53.9(6.3)$ & 0.112 \\
\hline $\begin{array}{l}\text { Marital status (Married/Co-habiting) } \\
\dagger\end{array}$ & $54.5(54)$ & $55.0(22)$ & 0.648 & $46.7(42)$ & $69.4(34)$ & 0.040 \\
\hline $\begin{array}{l}\text { Level of education (formal } \\
\text { education) } \dagger\end{array}$ & $94.9(94)$ & $97.5(39)$ & 0.978 & $95.6(96)$ & $95.9(47)$ & 0.766 \\
\hline Employed (Yes) † & $27.3(27)$ & $27.5(11)$ & 0.567 & $73.3(66)$ & $71.4(35)$ & 0.844 \\
\hline SES Quintile & $3.6(1.3)$ & $3.5(1.3)$ & 0.652 & $3.4(1.3)$ & $4.0(1.3)$ & 0.008 \\
\hline \multicolumn{7}{|l|}{ Housing density * } \\
\hline People/room & $\begin{array}{r}0.86 \\
(0.75)\end{array}$ & $\begin{array}{c}0.69 \\
(0.78)\end{array}$ & 0.065 & $0.79(0.75)$ & $0.83(0.88)$ & 0.220 \\
\hline People/bedroom & $\begin{array}{c}1.67 \\
(1.25) \\
\end{array}$ & $\begin{array}{r}1.37 \\
(1.00)\end{array}$ & 0.040 & $1.50(1.25)$ & $1.67(1.0)$ & 0.330 \\
\hline \multicolumn{7}{|l|}{ Behavioural } \\
\hline \multicolumn{7}{|l|}{ Diet * } \\
\hline $\begin{array}{l}\text { Fruit and vegetable intake } \\
\text { (servings/day) }\end{array}$ & $\begin{array}{c}1.29 \\
(1.00)\end{array}$ & $\begin{array}{l}1.14 \\
(0.00)\end{array}$ & 0.070 & $1.14(1.00)$ & $1.29(2)$ & 0.194 \\
\hline $\begin{array}{l}\text { Sugar sweetened beverages } \\
\text { (servings/day) }\end{array}$ & $\begin{array}{c}0.29 \\
(0.10)\end{array}$ & $\begin{array}{l}0.29 \\
(0.00)\end{array}$ & 0.010 & $0.29(0.00)$ & $0.29(1.00)$ & 0.131 \\
\hline \multicolumn{7}{|l|}{ Health-compromising } \\
\hline $\begin{array}{l}\text { Alcohol \& Tobacco use (both } \\
\text { current; Yes) }\end{array}$ & $8.1(8)$ & $45.0(18)$ & $<0.001$ & $18.9(17)$ & $18.4(9)$ & 0.972 \\
\hline \multicolumn{7}{|l|}{ Objectively-measured Sleep } \\
\hline \multicolumn{7}{|l|}{ Quantity } \\
\hline \multicolumn{7}{|l|}{ Sleep Time (minutes/day) } \\
\hline Total & $458(67)$ & $\begin{array}{r}456 \\
(106)\end{array}$ & 0.901 & $456(73)$ & $460(92)$ & 0.818 \\
\hline Nocturnal * & $434(61)$ & $411(72)$ & 0.054 & $425(60)$ & 431 (73) & 0.639 \\
\hline
\end{tabular}

Sufficient sleep categories $\dagger$

Total sleep time

\begin{tabular}{|c|c|c|c|c|c|}
\hline$<7$ hours/day & $32.3(32)$ & $40.0(16)$ & 0.666 & 31.1 (28) & $40.8(20)$ \\
\hline
\end{tabular}




$\begin{array}{lcccc}\text { 7-9 hours/day } & 52.5(52) & 45.0(18) & 56.7(51) & 38.8(19) \\ >9 \text { hours/day } & 15.2(15) & 15.0(6) & 12.2(11) & 20.4(10)\end{array}$

Nocturnal sleep time

\begin{tabular}{|c|c|c|c|c|c|}
\hline$<7$ hours/night & $45.5(45)$ & $50.0(22)$ & 0.546 & $45.6(41)$ & $53.1(26)$ \\
\hline 7-9 hours/night & $50.5(50)$ & $40.0(16)$ & & $52.2(47)$ & 38.8 (19) \\
\hline >9 hours/night & $4.0(4)$ & $5.0(2)$ & & $2.2(2)$ & $8.2(4)$ \\
\hline
\end{tabular}

Quality

Wake after sleep onset (minutes)

Sleep efficiency (\%)

$\begin{array}{cccc}54(16) & 44(17) & \mathbf{0 . 0 0 1} & 51(18) \\ 87.6 & 89.5 & \mathbf{0 . 0 1 7} & 88.2(4.6)\end{array}$

$51(15) \quad 0.839$

Achieved $\geq 85 \% \dagger$

$79.8(79)$

82.5 (33)

0.816

$80.0(72)$

$88.0(3.9)$

0.789

Activity counts during sleep (counts)

35057

(9223)

26.7

(7.0)

27448

0.001

32633

(9223)

Sleep fragmentation index (\%)

(9223)

$28.2 \quad 0.243$

26.5 (6.9)

(6.6)

\section{Objectively-measured Physical}

\section{Activity}

VM counts/day $\left(\mathrm{x} 10^{6}\right)$

$$
2.49
$$

2.00

$<0.001$

$2.33(0.75)$

$2.38(0.60)$

0.669

(0.63)

$(0.77)$

Ambulation (steps/day)

14288

15132

0.443

$14470(432)$

14681

0.838

(4378)

\section{Biological}

Females (Yes)

Waist circumference $(\mathrm{cm})$

93.0

(6492)

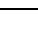

$65.6(59)$

$80.8<0.001$

$83.5(14.0)$

$81.6(40)$

0.052

(16.3)

(9.7)

30.4

(7.6)

21.7

$<0.001$

$25.3(6.9)$

$32.6(7.2)$

$<0.001$

(3.8)

Body mass index categories $\dagger$

Under- weight $\left(<18.5 \mathrm{~kg} / \mathrm{m}^{2}\right)$

$$
3.0 \text { (3) }
$$

17.5 (7)

$<0.001$

$10.0(9)$

$2.0(1)$

$<0.001$

Normal weight (18.5-24.9 kg/m²)

$21.2(21)$

$55.0(22)$

43.3 (39)

$8.2(4)$

$23.2(23)$

27.5 (11)

$24.4(22)$

$24.5(12)$

$52.5(52)$

$0.0(0.0)$

$22.2(20)$

$65.3(32)$

Conicity Index

$\begin{array}{ccc}1.23 & 1.23 & 0.940 \\ (0.10) & (0.08) & \end{array}$

HIV status (Yes) †

22.2 (22)

25.0 (10)

0.824

$126(24)$

0.870

126 (20)

78 (13)

0.157

94 (16) $\quad 0.384$
$1.20(0.09)$

28.9 (26)

120 (17)

77 (11)

91 (12)
12.2 (6)

0.034

138 (22) $<0.001$

86 (12) $<0.001$

MAP (mmHg) 


\begin{tabular}{|c|c|c|c|c|c|c|}
\hline Parity * $\ddagger$ & $4(2)$ & - & - & $4(2)$ & $4(3)$ & 0.775 \\
\hline \multicolumn{7}{|l|}{ Cardio-metabolic } \\
\hline \multirow[t]{2}{*}{ Total Cholesterol (mmol/l) } & 4.10 & 3.72 & 0.139 & $4.00(1.44)$ & $3.98(1.15)$ & 0.972 \\
\hline & $(1.36)$ & $(1.30)$ & & & & \\
\hline \multirow[t]{2}{*}{ HDL-Cholesterol (mmol/l) } & 1.10 & 1.07 & 0.865 & $1.16(0.53)$ & $0.95(0.34)$ & 0.007 \\
\hline & $(0.47)$ & $(0.52)$ & & & & \\
\hline \multirow[t]{2}{*}{ LDL-Cholesterol (mmol/l) } & 2.51 & 2.15 & 0.072 & $2.40(1.14)$ & $2.40(0.95)$ & 0.997 \\
\hline & $(1.10)$ & $(0.96)$ & & & & \\
\hline \multirow[t]{2}{*}{ Triglyceride (mmol/l) } & 1.10 & 1.10 & 0.959 & $0.95(0.47)$ & $1.38(0.68)$ & $<0.001$ \\
\hline & $(0.58)$ & $(0.61)$ & & & & \\
\hline \multirow[t]{2}{*}{ Glucose (mmol/l) } & 5.32 & 5.41 & 0.811 & $4.85(1.26)$ & $6.26(2.42)$ & $<0.001$ \\
\hline & $(1.89)$ & $(1.86)$ & & & & \\
\hline \multirow[t]{2}{*}{ Insulin $(\mu \mathrm{IU} / \mathrm{ml}) *$} & 6.91 & 3.14 & 0.001 & $4.12(6.27)$ & $7.60(7.05)$ & 0.001 \\
\hline & $(7.75)$ & $(5.61)$ & & & & \\
\hline \multirow[t]{2}{*}{ HOMA-IR * } & 1.51 & 0.71 & 0.003 & $0.90(1.53)$ & $2.09(1.96)$ & 0.003 \\
\hline & $(1.91)$ & $(1.72)$ & & & & \\
\hline \multicolumn{7}{|l|}{ HOMA-IR categories $†$} \\
\hline $\mathrm{Q} 1(\leq 0.82)$ & - & - & - & $42.2(38)$ & $16.3(8)$ & 0.002 \\
\hline Q2 (0.83 - 2.09) & - & - & & $33.3(30)$ & $34.7(17)$ & \\
\hline Q3 (2.10) & - & - & & $24.4(22)$ & $49.0(24)$ & \\
\hline \multicolumn{7}{|l|}{ Metabolic Syndrome (JIS } \\
\hline \multicolumn{7}{|l|}{ Harmonised definition) $\dagger$} \\
\hline No risk factor & $11.1(11)$ & $12.5(5)$ & 0.006 & - & - & - \\
\hline 1 risk factor & $15.2(15)$ & $42.5(17)$ & & - & - & \\
\hline 2 risk factors & $33.3(33)$ & $22.5(9)$ & & - & - & \\
\hline$\geq 3$ risk factors & $40.4(40)$ & $22.5(9)$ & & - & - & \\
\hline Waist circumference (male: $\geq 86 \mathrm{~cm}$, & $58.6(58)$ & $35.0(14)$ & 0.015 & $31.1(28)$ & $89.8(44)$ & $<0.001$ \\
\hline \multicolumn{7}{|l|}{ females: $\geq 92 \mathrm{~cm}$ ) } \\
\hline Blood pressure (SBP: $\geq 135 \mathrm{mmHg}$, & $42.4(42)$ & $50.0(20)$ & 0.266 & $27.8(25)$ & $75.5(37)$ & $<0.001$ \\
\hline \multicolumn{7}{|l|}{ DBP: $\geq 85$ mmHg) } \\
\hline HDL-Cholesterol (male:<1 mmol/, & $72.7(72)$ & $45.0(18)$ & 0.003 & $53.3(48)$ & $85.7(42)$ & $<0.001$ \\
\hline \multicolumn{7}{|l|}{ female<1.3 mmol/l) } \\
\hline Triglyceride $\geq 1.7 \mathrm{mmol} / \mathrm{l})$ ) & $15.2(15)$ & $7.5(3)$ & 0.276 & $5.6(5)$ & $26.5(13)$ & 0.001 \\
\hline Glucose ( $\geq 5.6 \mathrm{mmol} / \mathrm{l})$ & $19.2(19)$ & $27.5(11)$ & 0.362 & $11.1(10)$ & $40.8(20)$ & $<0.001$ \\
\hline Metabolic Syndrome z-score * & - & - & - & $-1.17(2.50)$ & $2.21(1.97)$ & 0.599 \\
\hline
\end{tabular}

Data reported as mean (SD), * median (IQR) or †\% (n); ‡low risk: $\mathrm{n}=59$, high risk: $\mathrm{n}=40$; DBP: Diastolic Blood Pressure; HOMA-IR: Homeostasis Model Assessment of Insulin Resistance; HIV: Human Immunodeficiency Virus; JIS: Joint Interim Statement; MAP: Mean Arterial Pressure = ₫DBP + ₫SBP; Nocturnal: 18h01 - 05h59; SES: SocioEconomic Status; SBP: Systolic Blood Pressure; VM: Vector Magnitude 
Page 12/16 
Table 2 Multivariate linear regression models for continuous cardio-metabolic measures

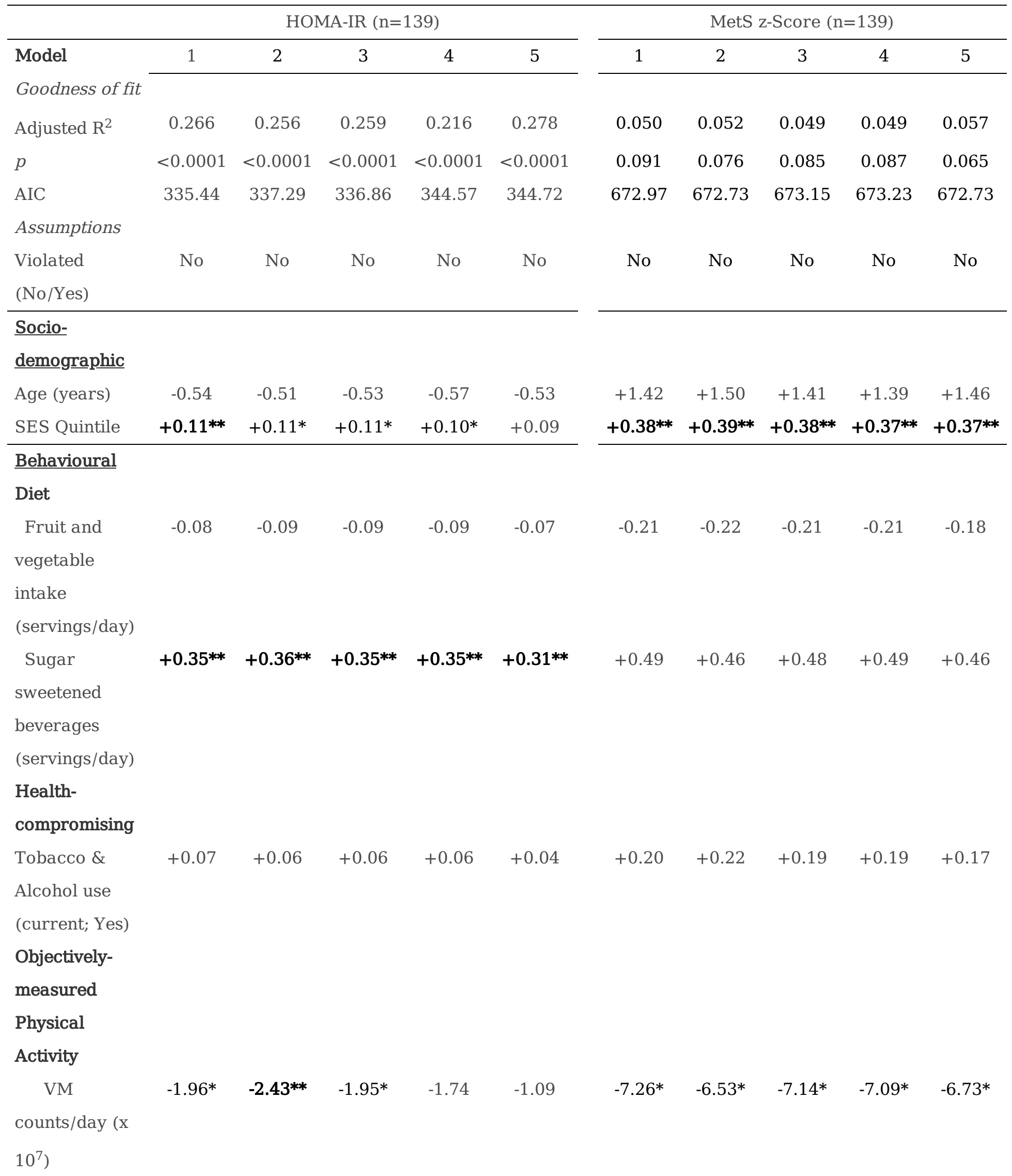

Objectively-

measured 
Sleep

Quantity

Sleep Time

(minutes/day)

$\begin{array}{lcccccccccc}\text { Total } & \mathbf{+ 1 . 0 0 * *} & - & +0.27 & +0.17 & +0.93 * & -1.60 & - & -1.97 & -2.01 & -0.99 \\ \text { Nocturnal } & - & +0.002 * & - & - & - & - & -0.004 & - & - & -\end{array}$

Quality

Sleep

$-0.07^{* *} \quad-0.62^{* *}$

efficiency (\%)

Wake after

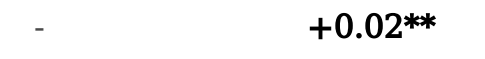

sleep onset

(minutes)

Activity counts

$+0.42^{* *}$

$-0.040$

$-0.04$

(counts during

sleep)

Sleep

$+0.03^{* *}$

$+0.04$

fragmentation

index (\%)

\section{Biological}

\begin{tabular}{|c|c|c|c|c|c|c|c|c|c|c|}
\hline $\operatorname{Sex}(0=\square, 1=\square)$ & $-0.31^{*}$ & $-0.31 *$ & $-0.30 *$ & $-0.33^{*}$ & $-0.43^{* *}$ & -0.24 & -0.30 & -0.25 & -0.26 & -0.33 \\
\hline Conicity Index & $+3.55^{* *}$ & $+3.38 * *$ & $+3.51 * *$ & $+3.41 * *$ & $+3.38 * *$ & - & - & - & - & - \\
\hline HIV status & -0.14 & -0.13 & -0.14 & -0.11 & -0.09 & $-1.13^{* *}$ & $-1.13^{* *}$ & $-1.13^{*}$ & $-1.11^{*}$ & $-1.15^{* *}$ \\
\hline
\end{tabular}

Data reported as $\pm \beta ; * 0.05 \leq \mathrm{p}<0.1, * * \mathrm{p}<0.05$; AIC: Akaike's Information Criterion; HIV: Human Immunodeficiency

Virus; HOMA-IR: Homeostasis Model Assessment of Insulin Resistance; MetS: Metabolic Syndrome; Nocturnal:

18 h01 - 05h59; SES: Socio-Economic Status; VM: Vector Magnitude

\section{Additional Files}

Additional File 1: Figure_S1.pdf

Figure S1 Cardiometabolic risk categories across categories of accelerometry-derived sleep-quantity and -quality measures.

A-C: Binary risk categories for the JIS Harmonised Definition

A. Total sleep time (minutes); B. Nocturnal sleep time (minutes); C. Sleep efficiency (percentage)

Fully-adjusted binary logistic regression models (socio-demographic, behavioural, biological), excluding body composition measures.

D-F: Tertiles of HOMA-IR

D. Total sleep time (minutes); E. Nocturnal sleep time (minutes); F. Sleep efficiency (percentage), ${ }^{*}$ OR $0.24(0.08,0.71)$ 
Fully-adjusted ordinal logistic regression models (socio-demographic, behavioural, biological), including body composition measures (Conicity Index).

The horizontal lines between the sleep parameter tertiles (Q1-Q3) indicate significant post hoc differences between tertiles at either end of the line.

\section{Additional File 2: Table_S1.docx}

Table S1. Cut-points for quantiles of sleep parameters.

\section{Figures}
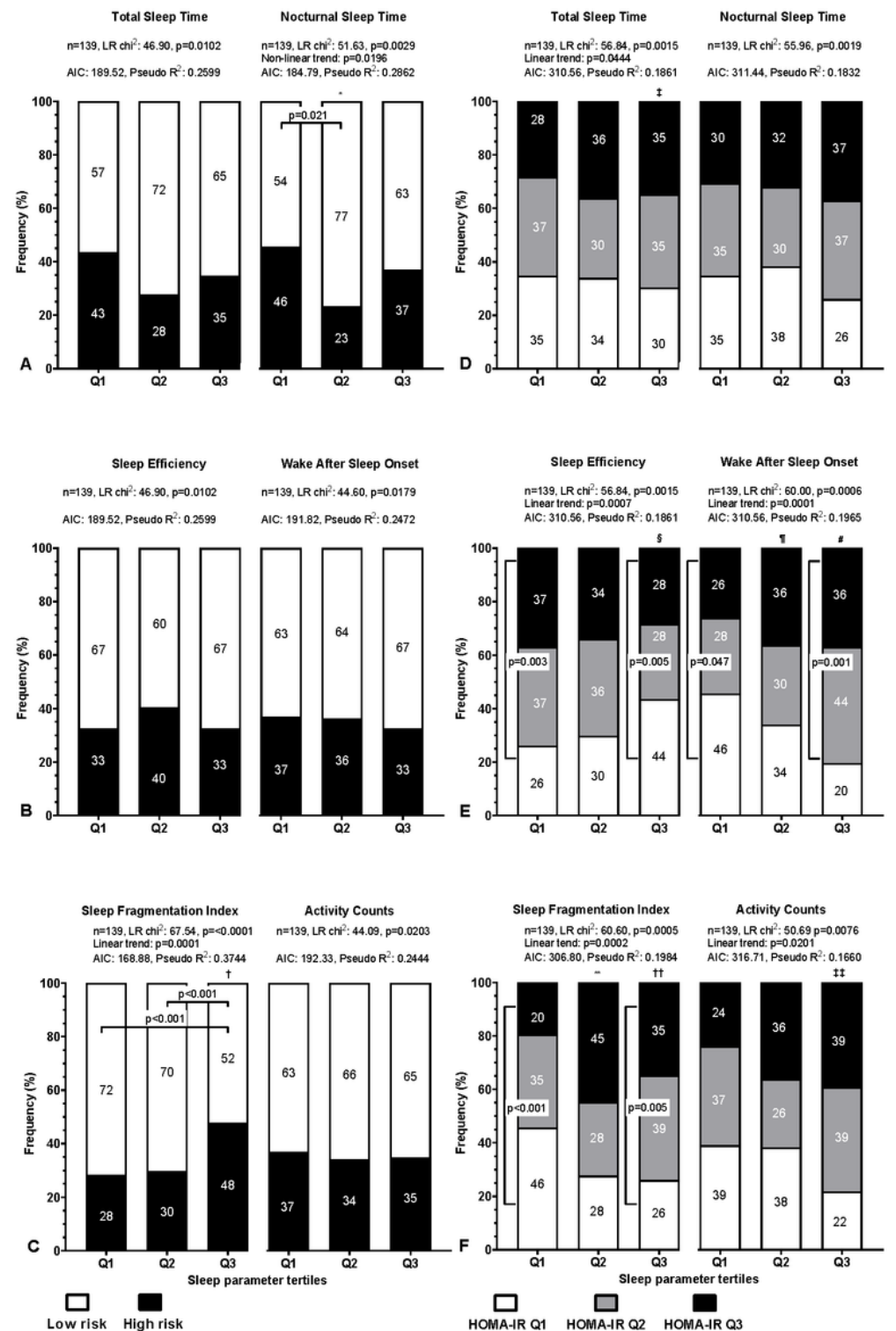

Figure 1

Cardiometabolic risk categories across quantiles of accelerometry-derived sleep-quantity and -quality measures. A-C: Binary risk categories for the JIS Harmonised Definition A. Nocturnal sleep time (minutes) and Total sleep time (minutes), * OR 0.20 (0.05, 0.71); B. Sleep efficiency (percentage) and Wake after sleep onset (minutes); C. Sleep fragmentation index (\%) and Activity counts during sleep (counts), tOR $69.80(8.44,577.63)$ Fully-adjusted binary logistic regression models (socio-demographic, behavioural, biological), 
excluding body composition measures. D-F: Tertiles of HOMA-IR D. Nocturnal sleep time (minutes) and Total sleep time (minutes), $¥$ OR 2.84 (1.03, 7.83); E. Sleep efficiency (percentage) and Wake after sleep onset (minutes), § OR 0.15 (0.05, 0.45), 9 OR 3.17 (1.21, 8.32), \# OR 6.75 (2.54, 17.91); F. Sleep fragmentation index (\%) and Activity counts during sleep (counts), ** OR 4.67 (1.64, 13.30$)$, †† OR 10.91 (3.11, 38.33), 㧊 OR 3.01 (1.19, 7.64) Fully-adjusted ordinal logistic regression models (socio-demographic, behavioural, biological), including body composition measures (Conicity Index). The horizontal lines between the sleep parameter tertiles (Q1-Q3) and vertical lines between the HOMA-IR tertiles (HOMA-IR Q1/Q2/Q3) indicate significant post hoc differences between tertiles at either end of the line. Cut-points for sleep parameter quantiles (Q1/Q2/Q3) are presented in Table S1 (see Additional file 2).

\section{Supplementary Files}

This is a list of supplementary files associated with this preprint. Click to download.

- FigureS1.pdf

- Tables1.docx 